\title{
Procedures for Estimating the Error in Padé Approximation
}

\author{
By Claude Brezinski
}

Dedicated to the memory of Professor Peter Henrici

\begin{abstract}
Kronrod's procedure is a method for estimating the error in Gaussian quadrature methods. Padé approximants are formal Gaussian quadrature formulas. In a previous paper, Kronrod's method was used to obtain estimates of the error in Padé approximation. Using a new interpretation of this procedure and three different expressions of the error of Padé approximants, extensions of the method are obtained. They provide new error estimates for Padé approximants. These estimates are compared.
\end{abstract}

1. Introduction. Kronrod's procedure [7] is a numerical method for estimating the error in Gaussian quadrature methods. On the other hand, Padé approximants can be viewed as formal Gaussian quadrature methods [2]. In [3], Kronrod's procedure was extended to Padé approximation to obtain estimates of the error. In this paper we shall give a new interpretation of this extension of Kronrod's method. This interpretation will lead to new procedures for estimating the error in Padé approximation.

Let us first recall some results on Padé approximants. Let $f$ be a formal power series

$$
f(t)=\sum_{i=0}^{\infty} c_{i} t^{i}
$$

and let $c$ be the linear functional on the space of complex polynomials defined by

$$
c\left(x^{i}\right)=c_{i}, \quad i \geq 0 .
$$

The functional $c$ can be extended to the space of formal power series, thus leading to formal identities.

Let $\left\{P_{k}\right\}$ be the family of formal orthogonal polynomials with respect to $c$, that is, defined by

$$
c\left(x^{i} P_{k}(x)\right)=0, \quad i=0, \ldots, k-1 .
$$

In the sequel it will always be assumed that the Hankel determinants $H_{k}\left(c_{0}\right)$ are different from zero. In that case, $P_{k}$ exists and has the exact degree $k$; the Padé approximant $[k-1 / k]_{f}$ also exists and the Padé table is normal. For nonnormality of the Padé table, see [5, Chapter 1]. Let $\left\{Q_{k}\right\}$ be the family of "associated" polynomials defined by

$$
Q_{k}(t)=c\left(\frac{P_{k}(x)-P_{k}(t)}{x-t}\right)
$$

Received June 22, 1987; revised October 5, 1988.

1980 Mathematics Subject Classification (1985 Revision). Primary 41A21, 41A25, 41A80. 
where $c$ acts on $x$ and $t$ is a parameter. $Q_{k}$ has the exact degree $k-1$. We set

$$
\tilde{P}_{k}(t)=t^{k} P_{k}\left(t^{-1}\right) \quad \text { and } \quad \tilde{Q}_{k}(t)=t^{k-1} Q_{k}\left(t^{-1}\right) .
$$

The rational function $\tilde{Q}_{k}(t) / \tilde{P}_{k}(t)$, denoted by $[k-1 / k]_{f}(t)$, is a Padé approximant of $f$. It satisfies the characteristic property

$$
f(t)-[k-1 / k]_{f}(t)=O\left(t^{2 k}\right) \quad \text { as } t \rightarrow 0 .
$$

More precisely, the error can be expressed in three different forms [2, Theorems 1.4 and 1.17]:

$$
\begin{aligned}
& \mathrm{E}_{1}: \quad f(t)-[k-1 / k]_{f}(t)=\frac{t^{k}}{\tilde{P}_{k}(t)} c\left(\frac{P_{k}(x)}{1-x t}\right), \\
& \mathrm{E}_{2}: \quad f(t)-[k-1 / k]_{f}(t)=\frac{t^{2 k}}{\tilde{P}_{k}(t)} c\left(\frac{x^{k} P_{k}(x)}{1-x t}\right), \\
& \mathrm{E}_{3}: \quad f(t)-[k-1 / k]_{f}(t)=\frac{t^{2 k}}{\tilde{P}_{k}^{2}(t)} c\left(\frac{P_{k}^{2}(x)}{1-x t}\right) .
\end{aligned}
$$

Let us also recall why Padé approximants can be viewed as "formal" Gaussian quadrature methods. We formally have

$$
f(t)=c\left(\frac{1}{1-x t}\right) .
$$

For obtaining an approximate value of $f(t)$, one can replace $(1-x t)^{-1}$ by an interpolation polynomial $P(x)$ and then compute $c(P(x))$. When $c$ is the integration functional, this is exactly the method followed in interpolatory quadrature formulas. Let $V_{n}$ be an arbitrary polynomial of degree $n$. The Hermite interpolation polynomial of $(1-x t)^{-1}$ at the zeros of $V_{n}$ (not necessarily all distinct) is given by

$$
P(x)=\left(1-V_{n}(x) / V_{n}\left(t^{-1}\right)\right) /(1-x t),
$$

since this is clearly a polynomial of degree at most $n$ and satisfies the interpolation conditions. It can be shown [2, Theorem 1.1] that $c(P(x))$ is the ratio of a polynomial of degree $n-1$ by a polynomial of degree $n$ and that

$$
f(t)-c(P(x))=O\left(t^{n}\right) .
$$

Such a rational function is called a Padé type approximant of $f$ and is denoted by $(n-1 / n)_{f}(t) . V_{n}$ is called the generating polynomial of $(n-1 / n)_{f}$. Furthermore $[2$, Theorem 1.4],

$$
(n-1 / n)_{f}(t)=\tilde{U}_{n}(t) / \tilde{V}_{n}(t)=f(t)-\frac{t^{n}}{\tilde{V}_{n}(t)} c\left(\frac{V_{n}(x)}{1-x t}\right)
$$

with

$$
U_{n}(t)=c\left(\frac{V_{n}(x)-V_{n}(t)}{x-t}\right), \quad \tilde{V}_{n}(t)=t^{n} V_{n}\left(t^{-1}\right) \quad \text { and } \quad \tilde{U}_{n}(t)=t^{n-1} U_{n}\left(t^{-1}\right) .
$$

If $V_{k}$ is identical with $P_{k}$, that is, if the interpolation points are the zeros of the orthogonal polynomial $P_{k}$, then $(k-1 / k)_{f}$ is identical with $[k-1 / k]_{f}$. This is exactly the method used to construct Gaussian quadrature methods and shows the connection with Padé approximation.

Kronrod's method [7] for estimating the error of a Gaussian quadrature formula consists in constructing a better quadrature formula by adding new nodes to the 
$k$ previous ones (the zeros of $P_{k}$ ) in an optimal way. Then the difference between both quadrature formulas provides an estimate of the error of the Gaussian one (see [6] for a review). This was exactly the method followed in [3] to extend Kronrod's procedure to Padé approximants. We shall now give a new interpretation of this procedure.

\section{Three Extensions.}

2.1. Extension Based on $\mathrm{E}_{1}$. Replace $(1-x t)^{-1}$ in $\mathrm{E}_{1}$ by its Hermite interpolation polynomial $P$ at the zeros of an arbitrary polynomial $V_{n}$. We set

$$
e_{k}^{(n)}=\frac{t^{k}}{\tilde{P}_{k}(t)} c\left(P_{k}(x) P(x)\right)
$$

It is easy to see, using (1.2), that

$$
e_{k}^{(n)}=t^{k} \tilde{W}_{n}(t) /\left[\tilde{P}_{k}(t) \tilde{V}_{n}(t)\right]
$$

with

$$
W_{n}(t)=c\left(P_{k}(x) \frac{V_{n}(x)-V_{n}(t)}{x-t}\right) \quad \text { and } \quad \tilde{W}_{n}(t)=t^{n-1} W_{n}\left(t^{-1}\right) .
$$

$\left(V_{n}(x)-V_{n}(t)\right) /(x-t)$ is a polynomial of degree $n-1$ in $x$. Thus, by the orthogonality properties of $P_{k}, W_{n}$ is identically zero if $n \leq k$ and the procedure has no interest.

Using $\mathrm{E}_{1}$ and (2.1), we have

$$
e_{k}^{(n)} /\left(f(t)-[k-1 / k]_{f}(t)\right)=c\left(P_{k}(x) /(1-x t)\right) \tilde{W}_{n}(t) / \tilde{V}_{n}(t) .
$$

But

$$
\begin{aligned}
\tilde{W}_{n}(t) & =t^{n-1} c\left(P_{k}(x) \frac{V_{n}(x)-V_{n}\left(t^{-1}\right)}{x-t^{-1}}\right) \\
& =\tilde{V}_{n}(t) c\left(\frac{P_{k}(x)}{1-x t}\right)-t^{n} c\left(\frac{P_{k}(x) V_{n}(x)}{1-x t}\right)
\end{aligned}
$$

and, by an old trick used by Stieltjes in his last letter to Hermite [1, Vol. 2, p. 439]:

$$
c\left(P_{k}(x) /(1-x t)\right)=c\left(\frac{1-t^{k} x^{k}+t^{k} x^{k}}{1-x t} P_{k}(x)\right)=t^{k} c\left(x^{k} P_{k}(x) /(1-x t)\right) .
$$

We have shown

THEOREM 1. There holds

$$
e_{k}^{(n)} /\left(f(t)-[k-1 / k]_{f}(t)\right)=1-\frac{t^{n-k}}{\tilde{V}_{n}(t)} \frac{c\left(P_{k}(x) V_{n}(x) /(1-x t)\right)}{c\left(x^{k} P_{k}(x) /(1-x t)\right)} .
$$

We remark that the computation of $e_{k}^{(n)}$ makes use of $c_{0}, \ldots, c_{n+k-1}$. As pointed out in [3], $e_{k}^{(n)}=(n+k-1 / n+k)_{f}(t)-[k-1 / k]_{f}(t)$, where the Padé type approximant $(n+k-1 / n+k)$ is constructed from the generating polynomial $v(x)=P_{k}(x) V_{n}(x)$. We have thus obtained an extension of Kronrod's procedure.

One can now try to choose $V_{n}$ in an optimal way, that is, achieving the best possible order of approximation. We have

$$
c\left(\frac{P_{k}(x) V_{n}(x)}{1-x t}\right)=c\left(P_{k}(x) V_{n}(x)\left(1+x t+\cdots+x^{n-1} t^{n-1}+\frac{x^{n} t^{n}}{1-x t}\right)\right) .
$$


Thus we have

THEOREM 2. If $V_{n}$ is chosen such that

$$
c\left(x^{i} P_{k}(x) V_{n}(x)\right)=0, \quad i=0, \ldots, n-1,
$$

then

$$
e_{k}^{(n)} /\left(f(t)-[k-1 / k]_{f}(t)\right)=1-\frac{t^{2 n-k}}{\tilde{V}_{n}(t)} \frac{c\left(x^{n} P_{k}(x) V_{n}(x) /(1-x t)\right)}{c\left(x^{k} P_{k}(x) /(1-x t)\right)}
$$

The computation of $e_{k}^{(n)}$ via Theorem 2 uses $c_{0}, \ldots, c_{2 n+k-1}$.

Since we shall take $n>k$, the smallest possible value is $n=k+1$ and then the method exactly reduces to Kronrod's procedure [3]. Of course, such a $V_{n}$ is assumed to exist, see [8].

In many practical applications, the coefficients $c_{i}$ of the series $f$ are difficult to compute. Thus, in this respect, Kronrod's method is expensive since the computation of $e_{k}^{(k+1)}$ needs the knowledge of $c_{0}, \ldots, c_{3 k+1}$ while that of $[k-1 / k]_{f}$ only requires $c_{0}, \ldots, c_{2 k-1}$. But, on the other hand, we obtain a rather good approximation of the error since

$$
e_{k}^{(k+1)} /\left(f(t)-[k-1 / k]_{f}(t)\right)=1+O\left(t^{k+2}\right), \quad t \rightarrow 0 .
$$

In order to reduce the cost of the method, it is possible to drop the condition on $V_{n}$ and to return to the nonoptimal procedure described by Theorem 1 , which only needs $c_{0}, \ldots, c_{n+k-1}$. Thus, one can make a compromise between the accuracy of $e_{k}^{(n)}$ and the number of coefficients needed.

We shall now study two other variants of the procedure used in Section 2.1.

2.2. Extension Based on $\mathrm{E}_{2}$. We now replace $(1-x t)^{-1}$ in $\mathrm{E}_{2}$ by its Hermite interpolation polynomial $P$ at the zeros of an arbitrary polynomial $V_{n}$.

We set

$$
e_{k}^{(n)}=\frac{t^{2 k}}{\tilde{P}_{k}(t)} c\left(x^{k} P_{k}(x) P(x)\right) .
$$

It is easily seen from (1.2) that

$$
e_{k}^{(n)}=t^{2 k} \tilde{W}_{n}(t) /\left[\tilde{P}_{k}(t) \tilde{V}_{n}(t)\right]
$$

with

$$
W_{n}(t)=c\left(x^{k} P_{k}(x) \frac{V_{n}(x)-V_{n}(t)}{x-t}\right) \quad \text { and } \quad \tilde{W}_{n}(t)=t^{n-1} W_{n}\left(t^{-1}\right)
$$

THEOREM 3. There holds

$$
e_{k}^{(n)} /\left(f(t)-[k-1 / k]_{f}(t)\right)=1-\frac{t^{n}}{\tilde{V}_{n}(t)} \frac{c\left(x^{k} P_{k}(x) V_{n}(x) /(1-x t)\right)}{c\left(x^{k} P_{k}(x) /(1-x t)\right)}
$$

Proof. Using $\mathrm{E}_{2}$ and (2.2), we have

$$
e_{k}^{(n)} /\left(f(t)-[k-1 / k]_{f}(t)\right)=c\left(x^{k} P_{k}(x) /(1-x t)\right) \tilde{W}_{n}(t) / \tilde{V}_{n}(t) .
$$


But, by definition,

$$
\begin{aligned}
\tilde{W}_{n}(t) & =t^{n-1} W_{n}\left(t^{-1}\right)=t^{n-1} c\left(x^{k} P_{k}(x) \frac{V_{n}(x)-V_{n}\left(t^{-1}\right)}{x-t^{-1}}\right) \\
& =\tilde{V}_{n}(t) c\left(\frac{x^{k} P_{k}(x)}{1-x t}\right)-t^{n} c\left(\frac{x^{k} P_{k}(x) V_{n}(x)}{1-x t}\right),
\end{aligned}
$$

and the result follows.

The computation of $e_{k}^{(n)}$ uses $c_{0}, \ldots, c_{2 k+n-1}$. Let us try to choose $V_{n}$ in an optimal way. We have

$$
c\left(\frac{x^{k} P_{k}(x) V_{n}(x)}{1-x t}\right)=c\left(x^{k} P_{k}(x) V_{n}(x)\left(1+x t+\cdots+x^{n-1} t^{n-1}+\frac{x^{n} t^{n}}{1-x t}\right)\right),
$$

and thus we immediately have the

THEOREM 4. If $V_{n}$ is chosen such that

$$
c\left(x^{i+k} P_{k}(x) V_{n}(x)\right)=0, \quad i=0, \ldots, n-1 \text {, }
$$

then

$$
e_{k}^{(n)} /\left(f(t)-[k-1 / k]_{f}(t)\right)=1-\frac{t^{2 n}}{\tilde{V}_{n}(t)} \frac{c\left(x^{n+k} P_{k}(x) V_{n}(x) /(1-x t)\right)}{c\left(x^{k} P_{k}(x) /(1-x t)\right)} .
$$

In this case the computation of $V_{n}$ needs $c_{0}, \ldots, c_{2 k+2 n-1} ; V_{n}$ is assumed to exist.

In the introduction of this paper we gave an interpretation of Kronrod's procedure which showed that it consisted in replacing $(1-x t)^{-1}$ by the interpolation polynomial $P$ in $\mathrm{E}_{1}$. We now give the reciprocal interpretation of this first extension.

Consider the Padé-type approximant $(2 k+n-1 / 2 k+n)_{f}$ with the generating polynomial $v(x)=x^{k} P_{k}(x) V_{n}(x)$. Let $w$ be its associated polynomial

$$
w(t)=c\left(\frac{v(x)-v(t)}{x-t}\right) .
$$

We have

$$
w(t)=W_{n}(t)+V_{n}(t) c\left(P_{k}(x) \frac{x^{k}-t^{k}}{x-t}\right)+V_{n}(t) t^{k} Q_{k}(t)
$$

where $W_{n}$ is defined immediately after (2.1) and $Q_{k}$ is defined in (1.1). Because of the orthogonality property of $P_{k}$, the second term on the right vanishes and we get

$$
\tilde{w}(t)=t^{2 k+n-1} w\left(t^{-1}\right)=t^{2 k} \tilde{W}_{n}(t)+\tilde{V}_{n}(t) \tilde{Q}_{k}(t) .
$$

Since $\tilde{v}(t)=t^{2 k+n} v\left(t^{-1}\right)=\tilde{P}_{k}(t) \tilde{V}_{n}(t)$, we obtain

$$
\frac{\tilde{w}(t)}{\tilde{v}(t)}=\frac{t^{2 k} \tilde{W}_{n}(t)}{\tilde{P}_{k}(t) \tilde{V}_{n}(t)}+\frac{\tilde{Q}_{k}(t)}{\tilde{P}_{k}(t)}
$$

and therefore, using (2.2),

$$
e_{k}^{(n)}=(2 k+n-1 / 2 k+n)_{f}(t)-[k-1 / k]_{f}(t) .
$$

Moreover, we have (cf. (1.3))

$$
f(t)-(2 k+n-1 / 2 k+n)_{f}(t)=\frac{t^{2 k+n}}{\tilde{P}_{k}(t) \tilde{V}_{n}(t)} c\left(\frac{x^{k} P_{k}(x) V_{n}(x)}{1-x t}\right) .
$$


If $V_{n}$ is chosen as in Theorem 4 , we have

$$
f(t)-(2 k+n-1 / 2 k+n)_{f}(t)=\frac{t^{2(k+n)}}{\tilde{P}_{k}(t) \tilde{V}_{n}(t)} c\left(\frac{x^{k+n} P_{k}(x) V_{n}(x)}{1-x t}\right) .
$$

2.3. Extension Based on $\mathrm{E}_{3}$. We now start from $\mathrm{E}_{3}$ and replace $(1-x t)^{-1}$ by the Hermite interpolation polynomial $P$. We set

$$
e_{k}^{(n)}=\frac{t^{2 k}}{\tilde{P}_{k}^{2}(t)} c\left(P_{k}^{2}(x) P(x)\right)
$$

Then, by (1.2),

$$
e_{k}^{(n)}=t^{2 k} \tilde{W}_{n}(t) /\left[\tilde{P}_{k}^{2}(t) \tilde{V}_{n}(t)\right]
$$

where now

$$
W_{n}(t)=c\left(P_{k}^{2}(x) \frac{V_{n}(x)-V_{n}(t)}{x-t}\right) \quad \text { and } \quad \tilde{W}_{n}(t)=t^{n-1} W_{n}\left(t^{-1}\right)
$$

Similarly as above, we can prove

THEOREM 5. There holds

$$
e_{k}^{(n)} /\left(f(t)-[k-1 / k]_{f}(t)\right)=1-\frac{t^{n}}{\tilde{V}_{n}(t)} \frac{c\left(P_{k}^{2}(x) V_{n}(x) /(1-x t)\right)}{c\left(P_{k}^{2}(x) /(1-x t)\right)} .
$$

If $V_{n}$ satisfies $c\left(x^{i} P_{k}^{2}(x) V_{n}(x)\right)=0$ for $i=0, \ldots, n-1$, then

$$
e_{k}^{(n)} /\left(f(t)-[k-1 / k]_{f}(t)\right)=1-\frac{t^{2 n}}{\tilde{V}_{n}(t)} \frac{c\left(x^{n} P_{k}^{2}(x) V_{n}(x) /(1-x t)\right)}{c\left(P_{k}^{2}(x) /(1-x t)\right)}
$$

In the first case, the computation of $e_{k}^{(n)}$ uses $c_{0}, \ldots, c_{2 k+n-1}$, in the second case $c_{0}, \ldots, c_{2 k+2 n-1}$. If we construct the Padé-type approximant $(2 k+n-1 / 2 k+n)_{f}$ with the generating polynomial $v(x)=P_{k}^{2}(x) V_{n}(x)$, then

$$
e_{k}^{(n)}=(2 k+n-1 / 2 k+n)_{f}(t)-[k-1 / k]_{f}(t) .
$$

The optimal $V_{n}$ is assumed to exist.

3. Comparisons. Of course, the three different approaches studied above are not independent. If the generating polynomials of the Padé-type approximants related to them are the same, then they provide the same estimate of the error.

In the first extension of the method, if we replace $n$ by $n+k$ and take $V_{n+k}(x)=$ $x^{k} V_{n}(x)$, where $V_{n}$ is the polynomial corresponding to the second extension, then both extensions are the same.

In the first extension, if $V_{n+k}(x)=P_{k}(x) V_{n}(x)$, where $V_{n}$ is the polynomial used in the third extension, then both extensions are the same.

In the preceding sections we gave three different methods for estimating the error in Padé approximation, each method having two possible versions: a general one where the polynomial $V_{n}$ was arbitrarily chosen, and an optimal one where $V_{n}$ was chosen such that $e_{k}^{(n)}$ was the best possible estimation of the error $f(t)-[k-1 / k]_{f}(t)$. For these six procedures we compare below the achieved order of approximation and 
the number of coefficients used in the computation of $e_{k}^{(n)}$. For the first extension we write $n^{\prime}$ instead of $n$.

$$
\text { order of approximation index of the last }
$$

First ext.

$$
\text { general } \quad n^{\prime}-k \geq 1 \quad n^{\prime}+k-1 \geq 2 k
$$
coefficient used

$$
\begin{array}{ccc}
\text { optimal } & 2 n^{\prime}-k \geq k+2 & 2 n^{\prime}+k-1 \geq 3 k+1 \\
\text { general } & n \geq 1 & 2 k+n-1 \geq 2 k
\end{array}
$$

Second ext.

$$
\begin{array}{lcc}
\text { optimal } & 2 n \geq 2 & 2 k+2 n-1 \geq 2 k+1 \\
\text { general } & n \geq 1 & 2 k+n-1 \geq 2 k
\end{array}
$$

Third ext.

$$
\text { optimal } \quad 2 n \geq 2 \quad 2 k+2 n-1 \geq 2 k+1
$$

Thus, in the general (nonoptimal) cases, the three procedures achieve the same order of approximation and use the same number of coefficients if $n^{\prime}=n+k$. In the optimal cases this will be true for $n^{\prime}=n+k / 2$. Since $n^{\prime} \geq k+1$ this can only happen when $n \geq k / 2+1 \geq 2$. If $n^{\prime}=2 n+k$, then the nonoptimal first extension and the optimal second and third extensions use the same coefficients and achieve the same order of approximation. If $2 n^{\prime}=k+n$, the optimal first extension and the nonoptimal second and third ones use the same coefficients and give the same order of approximation.

Let $e_{k}^{(n)}$ and $\bar{e}_{k}^{(n)}$ be two estimates of the error of $[k-1 / k]_{f}$. We define

$$
r(t)=\frac{e_{k}^{(n)} /\left(f(t)-[k-1 / k]_{f}(t)\right)-1}{\bar{e}_{k}^{(n)} /\left(f(t)-[k-1 / k]_{f}(t)\right)-1} .
$$

As stated in [3], if $|r(t)|<1$, then $e_{k}^{(n)}$ is a better estimate of the error than $\bar{e}_{k}^{(n)}$. Of course, if the order of $e_{k}^{(n)}$ is greater than the order of $\bar{e}_{k}^{(n)}$, then $\lim _{t \rightarrow 0} r(t)=0$ and $e_{k}^{(n)}$ will be a better estimate than $\bar{e}_{k}^{(n)}$ in a neighborhood of the origin. But when both estimates have the same order, this condition will be difficult to check. However, knowledge of $r(0)$ (whose computation uses some more coefficients of $f$ ) will provide some indication on this question. Using the preceding theorems, $r(0)$ is easy to compute.

We now show how to construct these estimates. We set

$$
\begin{aligned}
& V_{n}(x)=a_{0}+a_{1} x+\cdots+a_{n} x^{n}, \\
& P_{k}(x)=b_{0}+b_{1} x+\cdots+b_{k} x^{k}, \\
& e_{i}=c\left(x^{i} P_{k}(x)\right)=b_{0} c_{i}+\cdots+b_{k} c_{i+k}, \quad i=0,1, \ldots .
\end{aligned}
$$

Of course, $e_{i}=0$ for $i<k$.

(a) First Extension. We have

$$
\tilde{W}_{n}(t)=\sum_{i=0}^{n-1} \sum_{j=k+i+1}^{n} a_{j} e_{j-i-1} t^{n-i-1}, \quad n \geq k+1 .
$$


If $V_{n}$ is chosen in the optimal way, the $a_{i}$ 's must satisfy the system

$$
\sum_{j=k-i}^{n} a_{j} e_{i+j}=0, \quad i=0, \ldots, n-1,
$$

with $a_{n}=1$.

This system is triangular if and only if $n=k+1$, and in that case the procedure reduces to Kronrod's.

(b) Second Extension. We have

$$
\tilde{W}_{n}(t)=\sum_{i=0}^{n-1} \sum_{j=i+1}^{n} a_{j} e_{k+j-i-1} t^{n-i-1} .
$$

The optimal choice of $V_{n}$ leads to the system

$$
\sum_{j=0}^{n} a_{j} e_{i+k+j}=0, \quad i=0, \ldots, n-1,
$$

with $a_{n}=1$.

In general, this system is not triangular. It is easy to solve if $n=1$, in which case

$$
V_{1}(x)=x-e_{k+1} / e_{k} .
$$

(c) Third Extension. Let $d_{i}=c\left(x^{i} P_{k}^{2}(x)\right)$. Then

$$
d_{i}=\sum_{j=0}^{k} b_{j} e_{i+j}, \quad i=0,1, \ldots
$$

and

$$
\tilde{W}_{n}(t)=\sum_{i=0}^{n-1} \sum_{j=i+1}^{n} a_{j} d_{j-i-1} t^{n-i-1}
$$

The optimal choice of $V_{n}$ leads to the system

$$
\sum_{j=0}^{n} a_{j} d_{i+j}=0, \quad i=0, \ldots, n-1
$$

with $a_{n}=1$.

In general, this system is not triangular. For $n=1$ it gives

$$
V_{1}(x)=x-e_{k+1} / e_{k}-b_{k-1} / b_{k} .
$$

We conclude with a numerical example. Consider the series

$$
f(t)=t^{-1} \ln (1+t)=1-t / 2+t^{2} / 3-t^{3} / 4+\cdots .
$$

For $k=2$ we have $[1 / 2]_{f}=(6+3 t) /\left(6+6 t+t^{2}\right)$ and thus $P_{2}(x)=6 x^{2}+6 x+1$. For each of the three extensions, we shall compare three different choices of $V_{n}$ :

C1: $V_{n}(x)=x^{n} \quad$ which is an easy one and corresponds to the replacement of $(1-x t)^{-1}$ by $P(x)=1+x t+\cdots+x^{n-1} t^{n-1}$. We shall take $n^{\prime}=n+k$ in the first extension and $n$ in the two others.

C2: $V_{k}(x)=P_{k}(x) \quad$ which is also an easy choice but works only for the second and third extension.

C3: Optimal choice with $n=k / 2+1$ in the second and third extension and $k+1$ in the first (Kronrod's procedure). 
For the first extension with the first choice of $V_{n}$ we obtain

\begin{tabular}{|c|c|c|c|c|}
\hline$t$ & exact & $n^{\prime}=3$ & $n^{\prime}=5$ & $n^{\prime}=7$ \\
\hline 9 & 0.218002 & $0.15510710^{-1}$ & $0.57987710^{-1}$ & $0.96351610^{-1}$ \\
\hline 0.5 & $0.16789810^{-2}$ & $0.64102710^{-3}$ & $0.13965210^{-2}$ & $0.16111510^{-2}$ \\
\hline 0.1 & $0.72034910^{-6}$ & $0.61614510^{-6}$ & $0.71912810^{-6}$ & $0.72033910^{-6}$ \\
\hline 0.5 & $0.11940510^{-3}$ & $0.22522610^{-3}$ & $0.15283210^{-3}$ & $0.12769610^{-3}$ \\
\hline 0.9 & $0.64027210^{-3}$ & $0.17911610^{-2}$ & $0.18602510^{-2}$ & $0810^{-2}$ \\
\hline 2.0 & $0.38516010^{-2}$ & $0.24242510^{-1}$ & 0.11 & 70 \\
\hline 5.0 & $8610^{-1}$ & 0.341 & $17110^{2}$ & $0.31735510^{3}$ \\
\hline 7.0 & $0.18712610^{-1}$ & 0.82 & $0.61469010^{2}$ & $0.30936610^{4}$ \\
\hline
\end{tabular}

For this choice, $V_{n+k}(x)=x^{k} V_{n}(x)$, and the second extension will give the same results.

For the third extension and the first choice we have

\begin{tabular}{|c|c|c|c|}
\hline$t$ & $n=1$ & $n=3$ & $n=5$ \\
\hline-0.9 & $0.66002910^{-1}$ & 0.116071 & 0.143909 \\
\hline-0.5 & $0.11834410^{-2}$ & $0.15920010^{-2}$ & $0.16606810^{-2}$ \\
\hline-0.1 & $0.68334010^{-6}$ & $0.72011010^{-6}$ & $0.72034910^{-6}$ \\
\hline 0.5 & $0.14609210^{-3}$ & $0.12348310^{-3}$ & $0.12022210^{-3}$ \\
\hline 0.9 & $0.88017710^{-3}$ & $0.75570110^{-3}$ & $0.71445610^{-3}$ \\
\hline 2.0 & $0.66115910^{-2}$ & $0.10074910^{-1}$ & $0.23298510^{-1}$ \\
\hline 5.0 & $0.33593210^{-1}$ & 0.269549 & $0.49186010^{1}$ \\
\hline 7.0 & $0.51036410^{-1}$ & 0.825097 & $0.30209610^{2}$ \\
\hline
\end{tabular}

For the second choice, we get

\begin{tabular}{rcl}
\multicolumn{1}{c}{$t$} & second extension & third extension \\
-0.9 & $0.95704010^{-1}$ & 0.154474 \\
-0.5 & $0.14792910^{-2}$ & $0.16386010^{-2}$ \\
-0.1 & $0.71750710^{-6}$ & $0.71997010^{-6}$ \\
0.5 & $0.10957010^{-3}$ & $0.11845410^{-3}$ \\
0.9 & $0.48410210^{-3}$ & $0.62715510^{-3}$ \\
2.0 & $0.70934510^{-7}$ & $0.36063410^{-2}$ \\
5.0 & $-0.50388910^{-1}$ & $0.11565010^{-1}$ \\
7.0 & -0.127589 & $0.14206110^{-1}$
\end{tabular}

The optimal choices give

\begin{tabular}{rlll}
\multicolumn{1}{c}{$t$} & first extension & second extension & third extension \\
-0.9 & 0.218735 & 0.215560 & 0.184798 \\
-0.5 & $0.16794110^{-2}$ & $0.16792510^{-2}$ & $0.16754210^{-2}$ \\
-0.1 & $0.72034910^{-6}$ & $0.72035110^{-6}$ & $0.72035010^{-6}$ \\
0.5 & $0.11940910^{-3}$ & $0.11940810^{-4}$ & $0.11937610^{-3}$ \\
0.9 & $0.64039710^{-3}$ & $0.64031210^{-3}$ & $0.63927610^{-3}$ \\
2.0 & $0.38567510^{-2}$ & $0.38506310^{-2}$ & $0.38040310^{-2}$ \\
5.0 & $0.14156710^{-1}$ & $0.13901310^{-1}$ & $0.13099710^{-1}$ \\
7.0 & $0.18805410^{-1}$ & $0.18042710^{-1}$ & $0.16603910^{-1}$
\end{tabular}

4. Conclusions. As it can be seen from the preceding numerical examples, Kronrod's procedure provides the best possible choice of $V_{n}$ as stated by the theory. 
However, since such a choice needs the knowledge of many coefficients of the series, one may prefer a less efficient method using fewer coefficients.

Many open questions remain to be studied, for example the comparison between all the preceding possibilities and the convergence of the ratio $e_{k}^{(n)} /\left(f(t)-[k-1 / k]_{f}(t)\right)$ to one when $k$ and/or $n$ tend to infinity. In particular, if this is true for $k$ going to infinity, the sequence $\left([k-1 / k]_{f}(t)+e_{k}^{(n)}\right)$ will converge faster than $\left([k-1 / k]_{f}(t)\right)$ for the value of $t$ under consideration [4].

Acknowledgment. I would like to thank Professor W. Gautschi for his numerous suggestions for improving the presentation of this paper.

\author{
Laboratoire d'Analyse Numérique et d'Optimisation \\ Université des Sciences et Techniques \\ de Lille Flandres-Artois \\ 59655 Villeneuve d'Ascq-Cedex \\ France
}

1. R. Baillaud \& H. Bourget (Eds.), Correspondance d'Hermite et de Stieltjes, GauthierVillars, Paris, 1905.

2. C. Brezinski, Padé-type Approximation and General Orthogonal Polynomials, ISNM Vol. 50, Birkhäuser-Verlag, Basel, 1980.

3. C. BREZINSKI, "Error estimate in Padé approximation," in Orthogonal Polynomials and their Applications (M. Alfaro et al., eds.), Lecture Notes in Math., vol. 1329, Springer-Verlag, Heidelberg, 1988, pp. 1-19.

4. C. BREZINSKI, "A new approach to convergence acceleration methods," in Nonlinear $\mathrm{Nu}$ merical Methods and Rational Approximation (A. Cuyt, ed.), Reidel, Dordrecht, 1988, pp. 373-405.

5. A. DRAuX, Polynômes Orthogonaux Formels. Applications, Lecture Notes in Math., vol. 974, Springer-Verlag, Heidelberg, 1983.

6. W. GAU TSCHI, "Gauss-Kronrod quadrature-a survey," in Numerical Methods and Approximation Theory III (G. V. Milovanović, ed.), Nis, 1988, pp. 39-66.

7. A. S. KRONROD, Nodes and Weights of Quadrature Formulas, Consultants Bureau, New York, 1965.

8. M. PrÉvost, "Stieltjes and Geronimus-type polynomials," J. Comput. Appl. Math., v. 21, 1988, pp. 133-144. 